Regular Paper

\title{
Two-degree-of-freedom Control of a Piezoelectric Actuator with Hysteresis Compensation
}

\author{
M. SASAKI ${ }^{*}$, Y. ARIGA $*^{1}$, T. TAKEDA $*^{1}$ and S. ITO $*^{1}$
}

This paper presents the modeling and two-degree-of-freedom motion control of a piezoelectric bimorph actuator with hysteresis compensation. It has been demonstrated that with correctly applied voltage, the hysteresis effect can be substantially reduced. However, there still remains the problem of control. Two-degree-of-freedom control is a robust control technique that is effective for this actuator. It consists of a feed-forward controller based on an inverse system and a stabilizing feedback controller. Both experimental and numerical results are presented and show the effectiveness of both the control technique and the modeling method.

Keywords: piezoelectric actuator, applied electromagnetism, two-degree-of-freedom control, hysteresis compensation. (Received: 31 June 2012, Revised: 22 July 2013)

\section{Introduction}

This paper presents the modeling and two-degreeof-freedom motion control of a piezoelectric bimorph actuator with hysteresis compensation. There has been increasing interest in the modeling and control of piezoelectric actuator [1-5]. However, it is well-known that piezoelectric actuator suffer from nonlinearity due to hysteresis. At low drive inputs, hysteresis is not a big issue. At high drive levels however, the effect of hysteresis is profound. Hysteresis can cause loss of robustness, performance degradation and instabilities in feedback controlled piezoelectric actuators. Many techniques have been developed to combat the hysteresis associated with voltage controlled piezoelectric actuators. Inversion based precision modeling and phase controls are two examples of these techniques.

It has been argued that hysteresis is an electrical property of piezoelectric materials arising from the applied electric field and the resulting electrical charge. It has been shown that the hysteresis can be substantially reduced with the proper application of voltage. Even after compensating for hysteresis, the problem of control remains.

Two-degree-of-freedom control is a promising robust control technique for this actuator. Using a feedforward controller based on an inverse system and a stabilizing feedback controller, this technique is effective in suppressing the mechanical vibrations of a piezoelectric actuator and stabilizing it.

In this paper, we show results from the modeling and two-degree-of-freedom motion control of a piezoelectric bimorph actuator. Experimental and numerical results are presented, affirming the effectiveness of

\footnotetext{
Correspondence: M. SASAKI, Department of Human and Information Systems Engineering, Gifu University, 1-1 Yanagido, Gifu, 501-1193, Japan

email: sasaki@gifu-u.ac.jp

${ }^{* 1}$ Gifu University
}

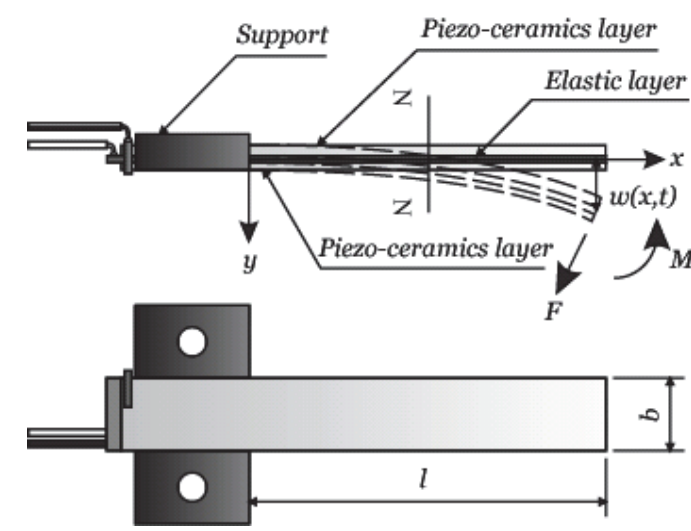

Fig. 1. Bimorph piezoelectric actuator.

both the modeling and control methods.

\section{System Modeling}

The piezoelectric actuator, provided by Fuji Ceramics, appears in Fig. 1. It is composed of a phosphor bronze central shim between two PZT flats with electrodes. Table 1 lists its material properties. Fig. 2 shows the experimental setup used to perform the bending tests described below.

The piezoelectric actuator was clamped horizontally between a pair of electrodes. When DC voltage is applied, the actuator bends towards the positive side. Vibration analysis was used to obtain a mathematical model of the relationship between input voltage and tip displacement. This was done by applying an AC voltage while monitoring tip displacement with a laser displacement meter and an FFT analyzer.

A step function of $8 \mathrm{~V}$ at time 1 second, shown in Fig. 3, was used to obtain the step response. From the step response a transfer function $G(s)$, shown in Eq. (1), between voltage and tip displacement was estimated. Using $G(s)$, a mathematical model of the PZT actuator was built. Based on the model, feed-forward and feedback controllers were designed and verified. Fig. 6 also shows good agreement between the experimental step response and the step response of $G(s)$. 
Fig. 7 shows the frequency characteristics of the PZT actuator obtained through vibration analysis. The first natural mode at $73 \mathrm{~Hz}$ is predominant.

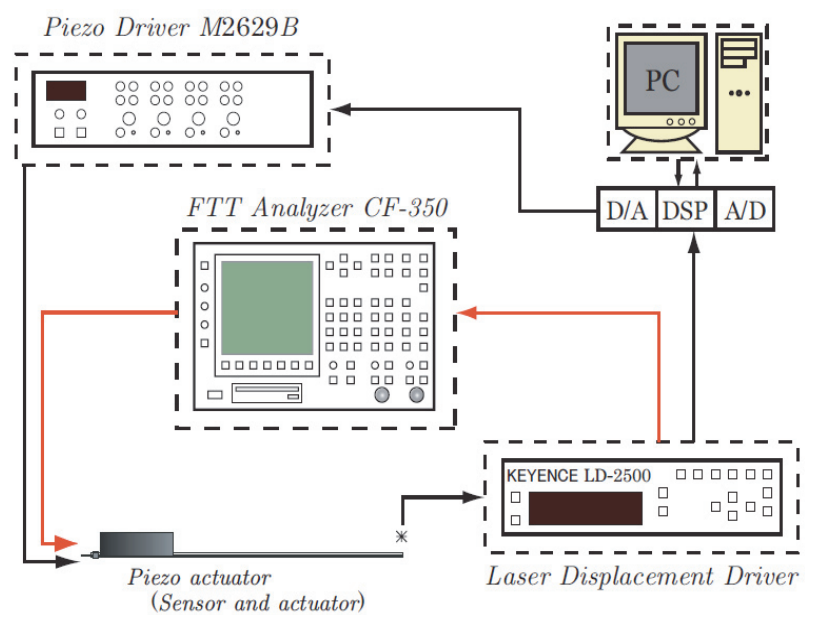

Fig. 2 Experimental setup.

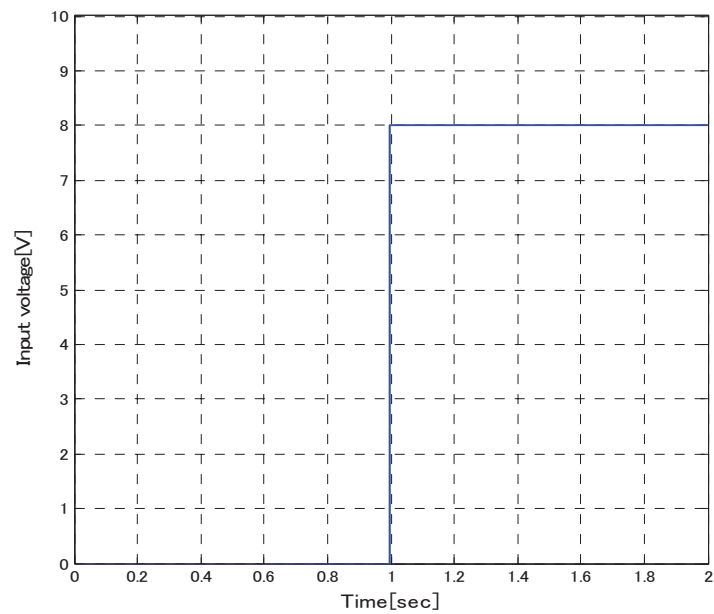

Fig. 3. Input voltage.

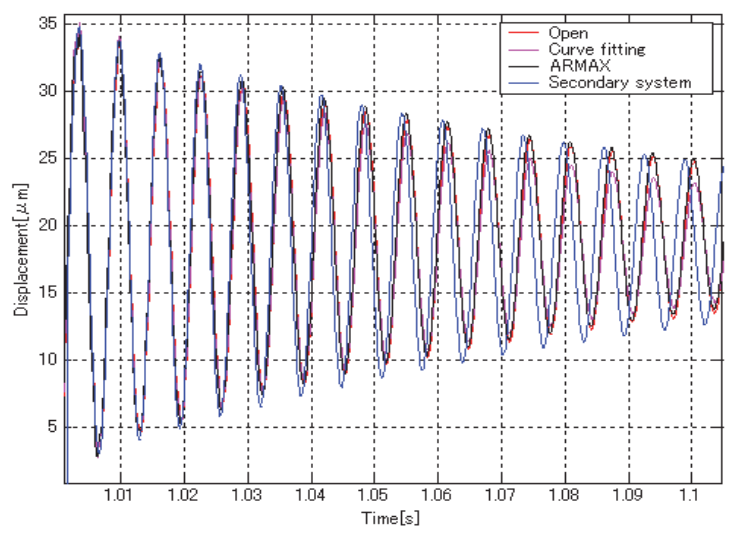

Fig. 4. Output displacement.
$G(s)=\frac{2.23 s^{2}+772.6 s+470530}{s^{2}+55 s+211000}$

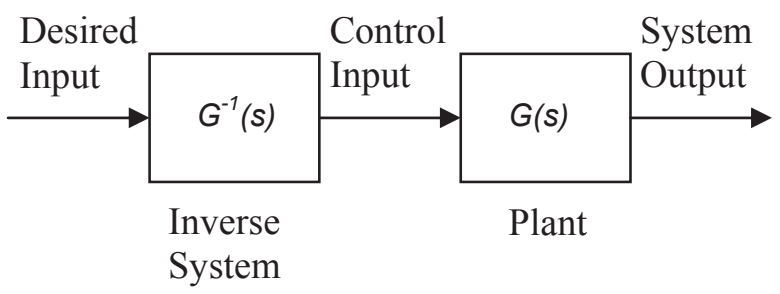

Fig. 5. Block diagram of the control system with the inverse system.

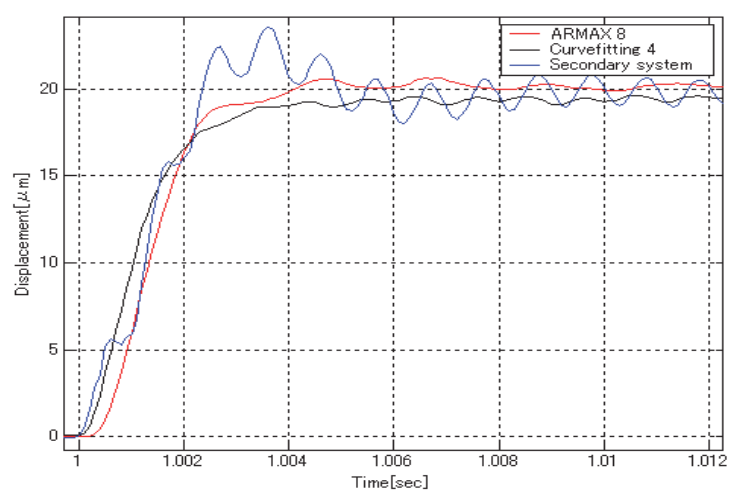

Fig. 6. Step response of the inverse system.

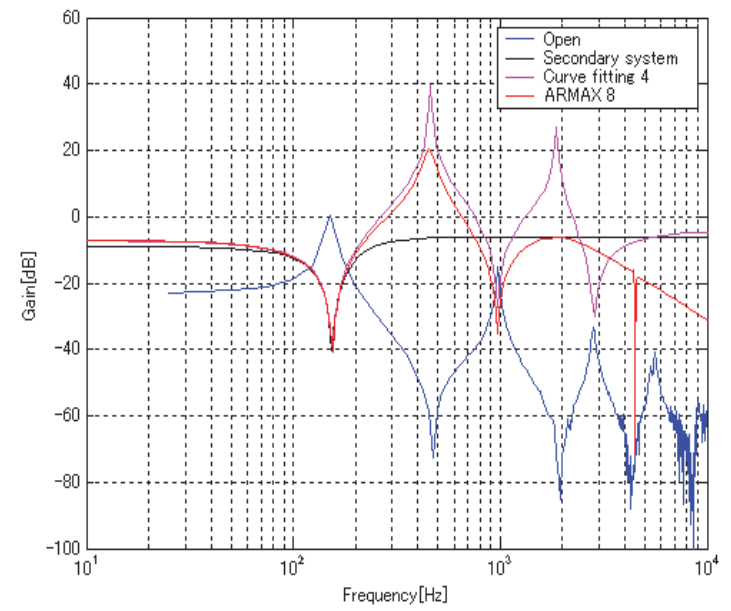

Fig. 7. Frequency response of the systems. 
Table. 1 Characteristic Data of the Piezoelectric Beam

\begin{tabular}{|c|c|c|c|}
\hline Symbol & Description & Value & Dimension \\
\hline$L$ & $\begin{array}{l}\text { Clamped Length of Piezoe- } \\
\text { lectric Beam }\end{array}$ & 51 & $m m$ \\
\hline$L_{-} t$ & $\begin{array}{l}\text { Total Length of Piezoelectric } \\
\text { Beam }\end{array}$ & 68 & $m m$ \\
\hline$h_{P}$ & $\begin{array}{l}\text { Thickness of Piezoelectric } \\
\text { Beam }\end{array}$ & 0.45 & $m m$ \\
\hline$h_{S}$ & $\begin{array}{l}\text { Thickness of Elastic Beam } \\
\text { (Shim) }\end{array}$ & 0.1 & $m m$ \\
\hline$h_{C}$ & $\begin{array}{l}\text { Thickness of Coating Mate- } \\
\text { rial (RTV Silicone) }\end{array}$ & 0.2 & $m m$ \\
\hline$b$ & Width of Beam & 10 & $m m$ \\
\hline$\rho_{P}$ & $\begin{array}{l}\text { Density of Piezoelectric } \\
\text { Material }\end{array}$ & $7.75 \times 10^{3}$ & $K g / m^{3}$ \\
\hline$\rho_{S}$ & Density of Shim Material & $8.2 \times 10^{3}$ & $K g / m^{3}$ \\
\hline$\rho_{C}$ & Density of Coating Material & $1.16 \times 10^{3}$ & $K g / m^{3}$ \\
\hline$E_{P}^{11}$ & $\begin{array}{l}\text { Young's Modulus of Piezoe- } \\
\text { lectric Material }\end{array}$ & $5.9 \times 10^{10}$ & Newton $/ m^{2}$ \\
\hline$E_{S}$ & $\begin{array}{l}\text { Young's Modulus of Shim } \\
\text { Material }\end{array}$ & $1.47 \times 10^{11}$ & Newton $/ m^{2}$ \\
\hline$E_{C}$ & $\begin{array}{l}\text { Young's Modulus of Coating } \\
\text { Material }\end{array}$ & $6.2 \times 10^{8}$ & Newton $/ m^{2}$ \\
\hline$d_{31}$ & Piezoelectric Constant & $-330 \times 10^{12}$ & $m /$ Volt \\
\hline$\in_{33}^{T}$ & $\begin{array}{l}\text { Relative Permittivty of } \\
\text { Piezoelectric Material }\end{array}$ & 5500 & Farad / $m$ \\
\hline$C p$ & $\begin{array}{l}\text { Total Capacitance of Piezoe- } \\
\text { lectric Material }\end{array}$ & 109.6 & nano Farad \\
\hline
\end{tabular}

\section{Inverse System}

Because the inverse of the model in Eq. (1) is improper, we use the low level passage inverse system $\hat{G_{w}}(s)$,

$$
G(s) \hat{G}_{w}(s)=\operatorname{diag}\left\{\frac{1}{(1+s T)^{\alpha}}, \cdots\right\} .
$$

In general, an inverse system has differentiating characteristics. However, an ideal differentiating element is, in principle, impossible, and an actual differentiating element would inevitably have the drawback of noise amplification in the high frequency range. This drawback is amended by reproducing the low frequency input only. Thus, low pass filtering was applied to derive an implementable inverse transfer function, shown in Eq. (3).

$$
G^{-1}(s)=\frac{s^{2}+55 s+211000}{2.23 s^{2}+772.6 s+470530}
$$

A block diagram of the feed-forward system appears in Fig. 5. It was implemented using the DSP subsystem in Fig. 2. Its step output is in Fig. 6 which shows good agreement between experimental and desired outputs. Its frequency response is included in Fig. 7. 


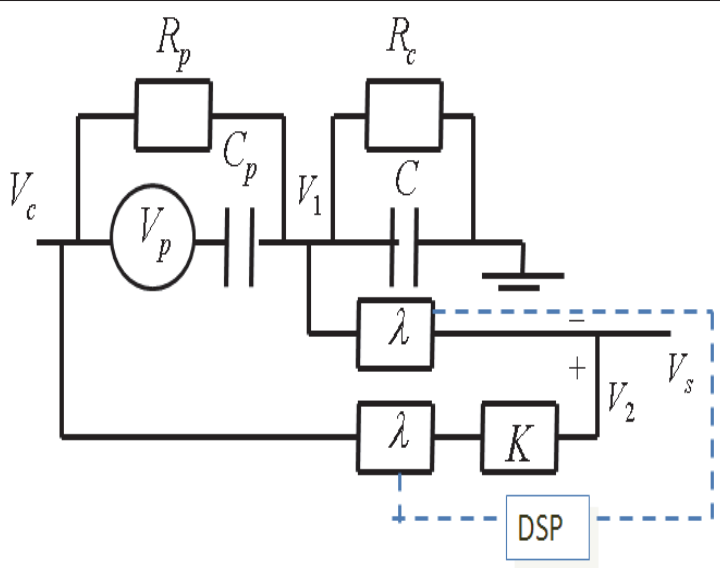

Fig. 8. Hysteresis compensation circuit system.

We used the same input as in Fig. 2 as the input of the inverse system and the strain history in Fig. 5 as the desired trajectory. Simulation results for the system with feed-forward control active for the same three regimes as in Fig. 2 are shown in Fig. 5. The feed-forward control does work with the inverse system.

Fig. 2 shows the experimental setup. The control input is calculated by the DSP (Texas Instruments) with a sample time of $0.01 \mathrm{msec}$ and applied to the bridge circuit through a D/A converter and a piezo driver. Sensor voltage $v_{S}$ from the bridge circuit is passed through a differential amplifier (Analog Devices, Precision Monolithic Instrumentation Amplifier) and then to the $\mathrm{A} / \mathrm{D}$ converter. A laser displacement sensor measures tip displacement.

Hysteresis has an adverse effect on the stability and closed loop performance of voltage controlled piezoelectric actuators. A large number of techniques have been developed to reduce hysteresis. It has been argued that hysteresis is an electrical property of piezoelectric materials arising from the applied electric field and the resulting electrical charge. It has been demonstrated that controlling electrical charge or current rather than the applied voltage substantially reduces the hysteresis effect. This method has not been widely used, perhaps due to the perceived difficulty of driving highly capacitive loads, which includes piezoelectric actuators. The main problem is the existence of offset voltages in the current source circuit which will eventually charge up the capacitive load. This will then distort the control signal. Fig. 8 shows the hysteresis compensation circuit. Fig. 9 shows the block diagram of the experimental system using a charge amplifier.

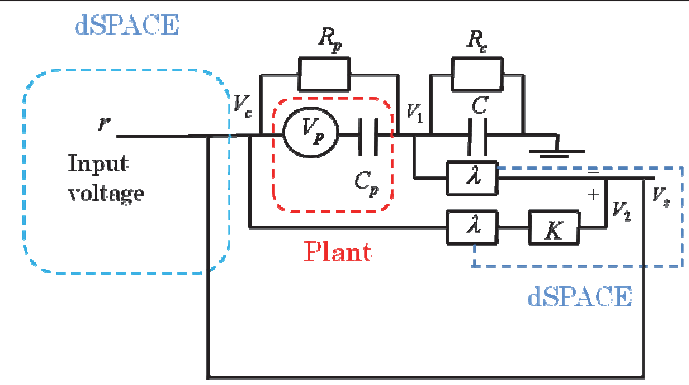

Fig. 9. Charge amplifier $\mathrm{C}$ bridge circuit.

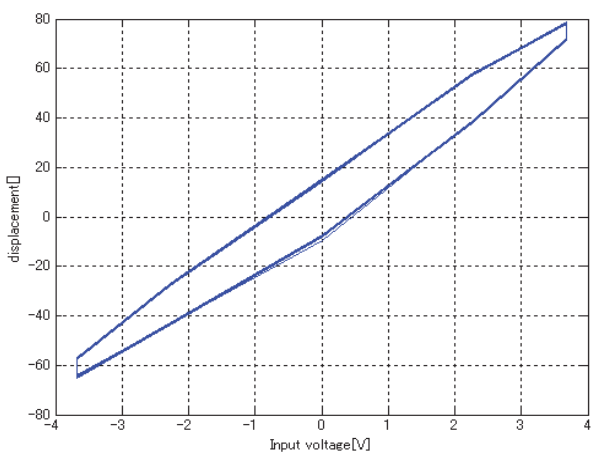

Fig. 10. Hysteresis curve of the actuator.

\section{Hysteresis Compensation}

The piezoelectric self-sensing actuator is a piezoelectric transducer used simultaneously as a sensor and an actuator. The key to this capability is knowing the capacitance of the piezoelectric transducer. Then, a voltage applied to the transducer can also be applied to an identically sized capacitor to obtain the capacitive electrical response. Subtracting this capacitive response from the response of the transducer yields the latter's mechanical response. A bridge circuit, described below, is used to isolate the piezoelectric strain signal.

Fig. 9 shows the bridge circuit of the selfsensing sensor. Voltage $v_{p}$ is the transducer output signal, consisting of the applied control signal and the piezoelectric response. In the Laplace domain, the self-sensing sensor voltage, $V_{1}-V_{2}$ is

$$
\begin{aligned}
& V_{1}-V_{2}=\frac{C_{p} R_{p} s}{1+C_{p} R_{p} s} V_{p} \\
& K=\frac{C_{p}}{C+C_{p}}
\end{aligned}
$$$$
C_{p}=100.4 \uparrow \mathrm{F}, C=1555 \multimap \mathrm{F}, R_{p}=100 \mathrm{M} \Omega,
$$
$R_{c}=6.531 \mathrm{M} \Omega, \lambda=-10$ and the cut-off frequency is $\omega_{c} \geq \frac{1}{C_{p} R_{p}}=0.143 \mathrm{~Hz}$. Fig. 11 shows the hysteresis compensation results with this bridge circuit, the uncompensated hysteresis curve shown in Fig. 10 has been eliminated. 


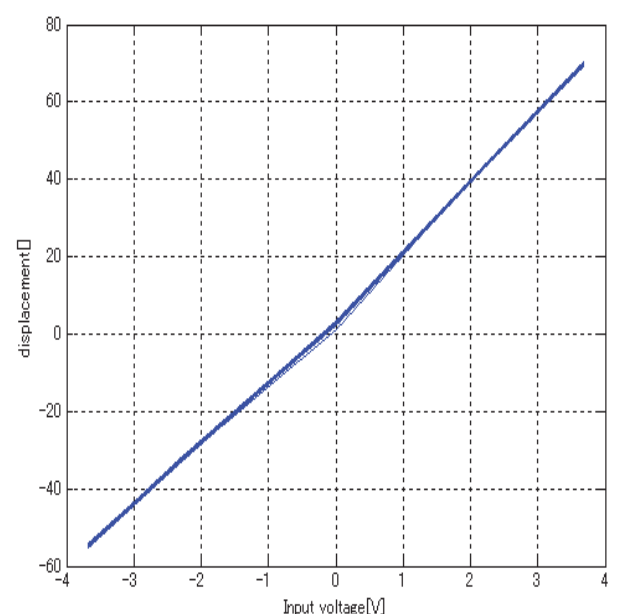

Fig. 11. Hysteresis curve of the actuator with hysteresis compensation.

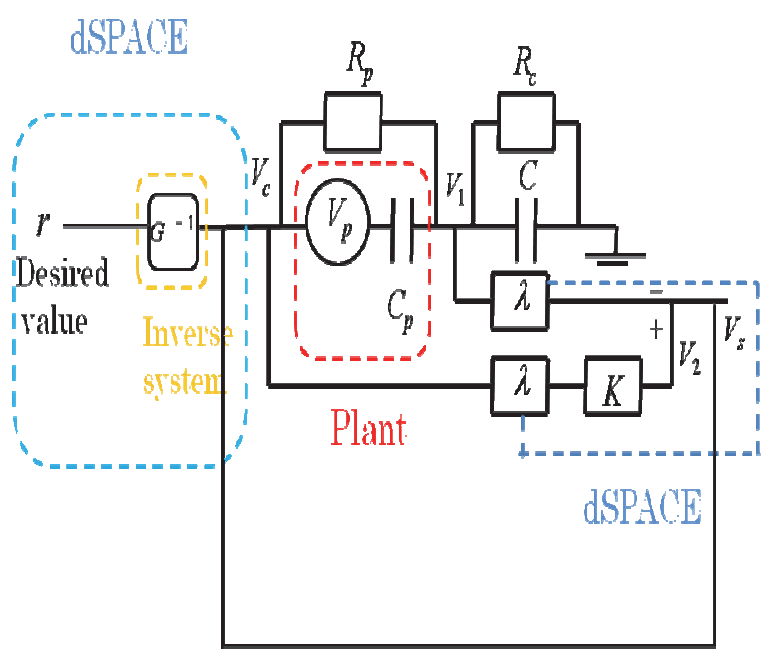

Fig. 12. Hysteresis compensation and inverse system.

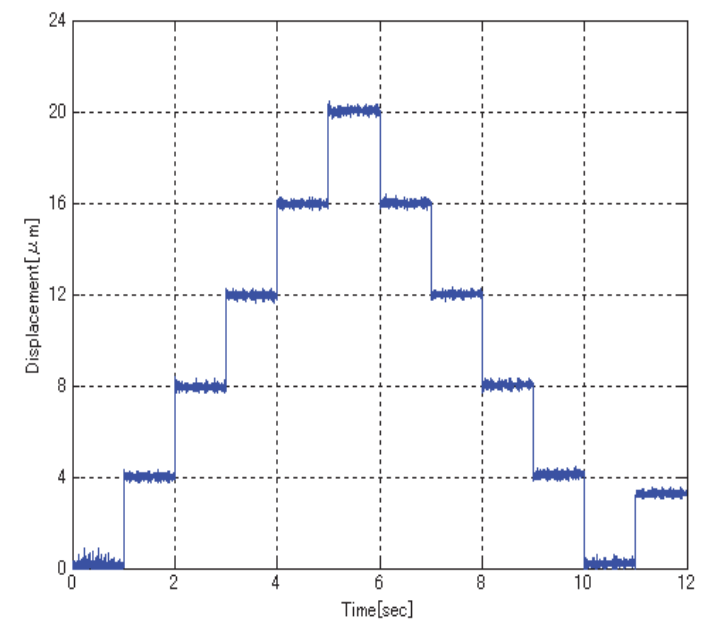

Fig. 13. Experimental result of hysteresis compensated, inverse system as shown in Fig. 12 with a disturbance at $11 \mathrm{sec}$.

\section{Control System Design.}

The controls engineer has many options available for many situations. Our approach to tracker design allows controller dynamics of any desired structure and then determines the control gains that minimize a quadratic PI over that structure. A dynamic compensator of prescribed nature may be incorporated into the system description as follows.

We begin with a familiar problem: the design of a controller to enable the tracking of a step reference input with zero steady-state error. The tracking error $e(t)$ is

$$
e=y-r
$$

Taking the time derivative yields

$$
\frac{d}{d t} e(t)=\mathbf{C} \dot{x}(t)
$$

The dynamic and output equations may be written in augmented form as

$$
\frac{d}{d t}\left[\begin{array}{l}
\dot{x}(t) \\
e(t)
\end{array}\right]=\left[\begin{array}{ll}
\mathbf{A} & 0 \\
\mathbf{C} & 0
\end{array}\right]\left[\begin{array}{l}
\dot{x}(t) \\
e(t)
\end{array}\right]+\left[\begin{array}{l}
\mathbf{B} \\
0
\end{array}\right] \dot{u}(t)+\mathbf{D} r
$$

where

$$
\begin{array}{ll}
\mathbf{A}=\left[\begin{array}{cc}
-23.24 & -9.376 \times 10^{5} \\
1 & 0
\end{array}\right] & \mathbf{B}=\left[\begin{array}{l}
1 \\
0
\end{array}\right] \\
\mathbf{C}=\left[\begin{array}{ll}
2203.5 & 7.4356 \times 10^{5}
\end{array}\right] & \mathbf{D}=[2]
\end{array}
$$

If the system is controllable, we can find a feedback of the form

$$
\dot{u}(t)=-F_{1} \dot{x}(t)-F_{2} e(t)
$$

such that Eq. (8) is stable, implying that tracking error $e(t)$ is stable. We will have achieved the objective of asymptotic tracking with zero steady-state error. The control input, found by integrating Eq. (9), is

$$
u(t)=-F_{1} x(t)-F_{2} \int_{0}^{t} e(t) d t
$$

We see that the compensator includes an internal model of the reference step input. However, this control system cannot be implemented unless all states are available as measurements, which is usually not the case.

Suppose that an observer Eq. (11) has been designed, with the filter gain $\mathrm{L}$ having been selected by any one of many techniques.

$$
\dot{\hat{x}}^{\prime}=\left(A^{\prime}-L C^{\prime}\right) \hat{x}^{\prime}+B u+L y
$$

Then, since all the states are not measurable, we propose implementing the control by feeding back the estimated state $\hat{x}(t)$ instead of the actual state $x(t)$. The feedback law becomes

$$
u(t)=-K_{1} \hat{x}(t)-K_{2} \int_{0}^{t} \hat{e}(t) d t
$$

The closed-loop structure using this controller is shown in Fig. 14. $K$ is selected using the LQR Riccati equation and $L$ is selected using the Observer Riccati 


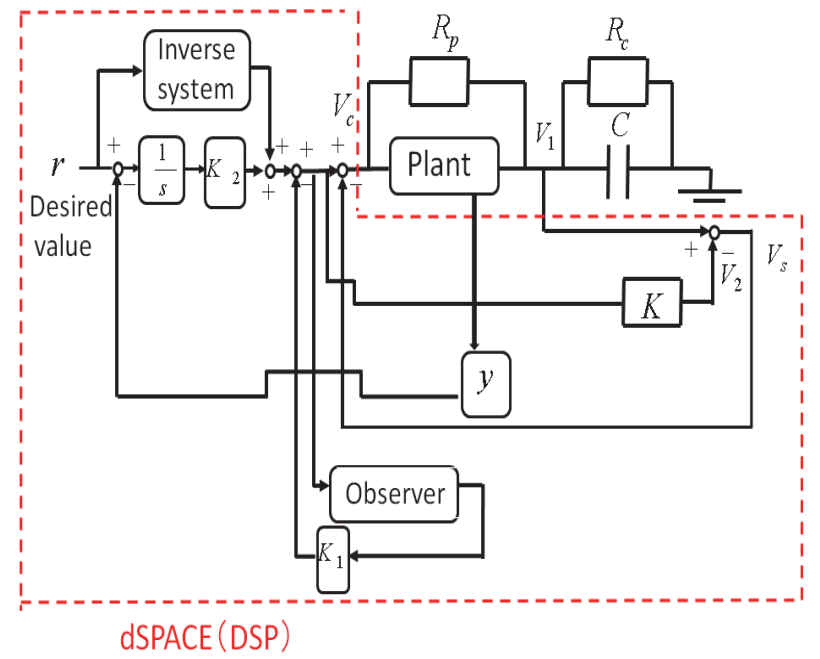

Fig. 14. Block diagram of the two-degrees-of-freedom control system with hysteresis compensation.

equation. The importance of these results is that the tracking feedback $K$ and the observer gain $L$ can be designed separately to yield desired closed loop plant and observer behavior.

\section{Experimental Results and Discussion.}

After several design cycles, we settled on these values for the weighting parameters $R$ and $Q$,

$$
R=0.01, Q=\operatorname{diag}[100010100] \text {. }
$$

With these values, we obtain the gains

$$
\begin{aligned}
& K_{1}=\left[\begin{array}{ll}
-0.0029 \times 10^{5} & 1.7056
\end{array}\right], K_{2}=[100.00], \\
& L=\left[\begin{array}{ll}
0.0081352 .7883 \times 10^{-6}
\end{array}\right] .
\end{aligned}
$$

Fig. 15 shows the experimental step response using the above designed controller with a sampling time of $0.001 \mathrm{sec}$. The controller successfully stabilizes the actuator close to the desired position. There is good agreement between simulated and actual results. The methods we used are validated. Comparison between Fig. 13 and Fig. 14, the feedforward controller can track the desired trajectory without a disturbance. The twodegree-of-freedom controller can track the desired trajectory and compensate the disturbance after $11 \mathrm{sec}$.

\section{Conclusion.}

This paper has presented the modeling and motion control of a piezoelectric bimorph actuator, overcoming hysteresis in the process. The flexible motions of the actuator were stabilized and control was achieved. The experimental and simulation results compare well, demonstrating the effectiveness of the methods used. The two-degree-of freedom control system has the robustness for the step disturbance.

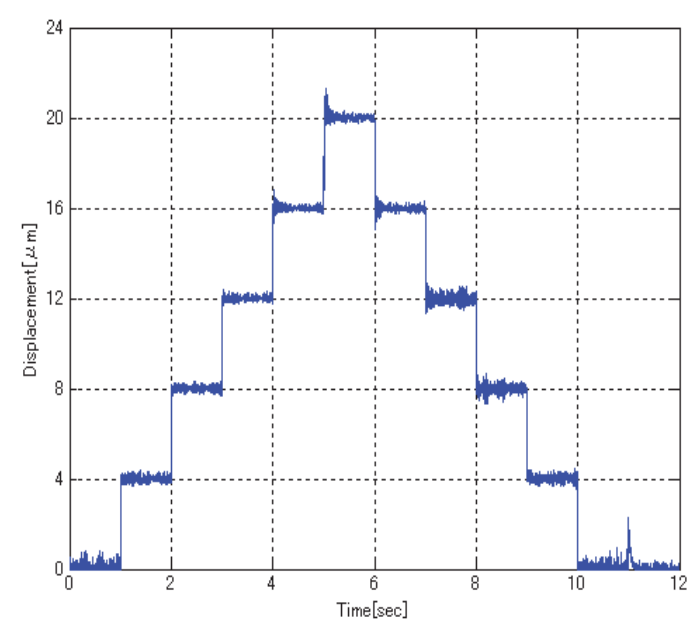

Fig.15. Experimental results of hysteresis compensated, two-degree-of-freedom control with a disturbance at $11 \mathrm{sec}$.

\section{References}

[1] H. Yamada, M. Sasaki and Y. Nam, "Control of a MicroActuator for Hard Disk Drives Using Self-Sensing Actuator," Int. J. Appl. Electromagn. and Mech., Vol. 25, No. 1, 2007.

[2] Y. Nam, J. Yoon and M. Sasaki, "End Point Motion Estimation of a Cantilevered Piezoelectric Beam," JSME Int. J. Series C: Mechanical Systems, Machine Elements and Manufacturing, Vol. 45, No. 3, pp. 722729, 2002.

[3] H. Yamada, M. Sasaki and Y. Nam, "Control of a Microactuator for Hard Disk Drives Using Self-Sensing Actuator," Int. J. Appl. Electromagn. Mech., Vol. 24, No. 4, pp. 247-259, 2006.

[4] H. Yamada, M. Sasaki and Y. Nam, "Active Vibration Control of a Micro-Actuator for Hard Disk Drives Using Self-Sensing Actuator," J. Intelligent Mater. Sys. and Structures, Vol. 19, pp. 113-123, 2008.

[5] M. Sasaki, K. Fujihara, H. Yamada, Y. Nam, S. Ito and Y. Inoue, "Two-Degree-of-Freedom Control of a SelfSensing Micro-Actuator for HDD," Int. J. Appl. Electromagn. And Mech., Vol. 36, pp.159-166, 2011. 